\title{
An Iris Localization Algorithm based on Geometric Features of the Circle
}

\author{
Ming Fei Wang ${ }^{1}$, RuiYun Xie ${ }^{1}$ and BenZhai Hai ${ }^{2}$ \\ 1. Computer Science and Technology Department, Henan Mechanical and \\ Electrical \\ Engineering College, Henan Xinxiang 453000, China \\ 2. Information Engineering College, Wuhan University Of Technology, Hubei \\ Wuhan 430070, China \\ 421856163@qq.com
}

\begin{abstract}
An iris localization algorithm with the fast speed is proposed based on geometric features of the circle combing coarse localization with fine localization to avoid the problem of the slow speed of the classical iris localization algorithm. First, the row and column scanning method is employed to find four strings in the pupil. Four couples of tangency points are located by using the perpendicular bisector of strings and the threshold of the pupil boundary. The mean value of the coordinate parameters of the tangency points is regarded as the parameters for inner edge rough localization parameters. Then the calculus method is used for precise localization of the inner edge. The priori knowledge of the close distance between inner and outer edge centers of the circle is used to narrow the search range of the calculus method localizing outside edge, to significantly improving the speed of iris localization. The experimental results show that the proposed algorithm can improve the speed of iris localization with the high localizing accuracy.
\end{abstract}

Keywords: iris location, geometric feature of the circle, calculus method, Hough transform

\section{Introduction}

Iris localization refers to a localization of the inner edge of the iris (the pupil edge) and the iris outer boundary. As in the phase of acquiring images, the images usually contain the whole eye area, it is necessary to separate the iris from the eye image, which is the key step of iris recognition system. The accurate iris localization is a precondition to realize iris verification and identification. Iris localization is usually divided into the following three steps: smooth denoising of iris images, determining the center and the radius of the circle of the inner edge of the iris, and determining the center and radius of the circle of the iris outer edge. Current iris localization algorithms are mainly divided into two categories: one is the calculus method based on iris edge gradient represented by Daugman [1], and the other is Hough transform method represented by Wildes [2]. Besides, there are some localization methods based on geometric features.

\subsection{The Calculus Method}

Daugman believes that the grayscale distributions of the iris image of the acquisition have some differences. In general, the iris is darker than the sclera, while the pupil is darker than the iris. Therefore, the grayscale gradient is formed obviously in the iris edge. According to the prior knowledge of the iris shape being similar to the annular, Daugman used the detection operator to localize the inner and outer edge of the iris. The core of this 
algorithm is to employ an effective differential operator to calculate the iris edge parameter [3]:

$$
\max _{\left(r, x_{0}, y_{0}\right)} \mid G_{\sigma}(r) * \frac{\partial}{\partial r} \oint_{\left(r, x_{0}, y_{0}\right)} \frac{I(x, y)}{2 \pi r} d s
$$

In the above formula:

* - The convolution operation

$G_{\sigma}(r)-$ Gaussian function is used to smooth the image

$r-$ The radius of the circle detection operator

$I(x, y) \longrightarrow$ The grayscale value of the iris image in the point $(\mathrm{x}, \mathrm{y})$.

In the formula (1), Gaussian function is shown:

$$
G_{\sigma}(r)=(1 / \sqrt{2 \pi} \sigma) e^{-\left(r-r_{0}\right)^{2} / 2 \sigma^{2}}
$$

In the formula (2): $r_{0} \longrightarrow$ the center of Gaussian function

$\sigma-$ Standard deviation of Gaussian function

The physical meaning of formula (1) is to search for changes with circular detection operator radius, and the corresponding pixel grayscale value changes on the circumference of the average maximum values of data $\left(r, x_{0}, y_{0}\right)$. It is regarded as the parameters of the iris inner and outer edge. Convolution is used to smooth the image and eliminate the influence of noise in iris image. The size of the smooth template is related to the precision of the localization. In order to get the discrete implementation of formula (1), the character of the convolution is used to transform the formula (1) into:

$$
\max _{\left(r, x_{0}, y_{0}\right)}\left|\left(\frac{\partial G_{\sigma}(r)}{\partial r}\right) * \oint_{\left(r, x_{0}, y_{0}\right)} \frac{I(x, y)}{(2 \pi r)} d s\right|
$$

To make the algorithm into practice in the computer, it is necessary to do discrete processing, and the finite difference of the discrete sequence $n$ is used as effective approximate differential, and the formula is shown:

$$
\frac{G_{\sigma}(r)}{\partial r} \approx G_{\sigma}(n)=\frac{1}{\Delta r} G_{\sigma}(n \Delta r)-\frac{1}{\Delta r} G_{\sigma}((n-1) \Delta r)
$$

In the above formula: $\Delta r-$ The search step of the radius.

The formula (3) is discretized, and cumulative sum of $\Sigma$ are used instead of convolution integral and curve integral, and the formula is converted as:

$$
\max _{\left(n \Delta r, x_{0}, y_{0}\right)}\left|\frac{1}{\Delta r} \sum_{k}\left\{G_{\sigma}(r) \sum_{m} I(x(m, k), y(m, k))\right\}\right|
$$

In the above formula : $G_{\sigma}(r)=G_{\sigma}((n-k) \Delta r)-G_{\sigma}((n-k-1) \Delta r)$

$x(m, k), y(m, k)$ are given in the following formula:

$$
\left\{\begin{array}{l}
x(m, k)=\left(k \Delta r \cos (m \Delta \theta)+x_{0}\right) \\
y(m, k)=\left(k \Delta r \sin (m \Delta \theta)+y_{0}\right)
\end{array}\right.
$$

In the above formula: $\Delta \theta \longrightarrow$ the step length of the separated angle along the network arc . 
Because the Daugman's iris images are mostly from western populations, the gray scale gradient of the iris outer circle of iris images collected by him is large with a clear edge. When he used the calculus method to localize the inner and outer edge of the iris, iris outer circle is localized at first, and then according to the feature of the circle center of the pupil edge near the iris the outer circle, the iris inner circle is localized [4]. Formula (1) is also used to localize the upper and lower eyelids, but the integral path changes from round into a section of circular arc. Results of the calculus operator are used to localize iris as shown in figure 1.

Advantages of Daugman localization algorithm are very high robustness and accuracy, even though the iris image with the blur edge and low contrast can be localized well. The shortage of the Daugman localization algorithm is that the algorithm uses gradient magnitude information, which is easily influenced by the local gradient caused by the light source and other factors to lead to the localization failure. In terms of the localization speed, if the range of parameters is not limited, in the whole image, the two parameters of the center and the radius do the iterative search, which will be very time-consuming [5].

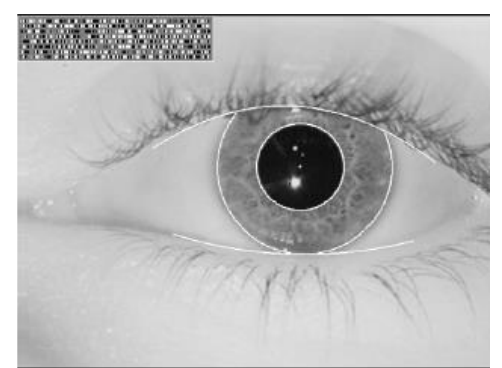

\section{Figure 1. Iris Edge Localization based on the Calculus Algorithm}

\subsection{Hough Transform Method}

Wildes used the Hough transform to detect the inner and outer edge of the iris. Wildes has taken two steps in iris boundary localization: in the first step, the grayscale image is transferred into binaryzation edge image; in the second step, the vote is done for the edge points to obtain the parameters of the inner edge and the outer edge of the iris [6].

Step one: Two-value edge image is obtained based on the gradient edge detection operator. This operator is defined as follows:

$$
|\nabla G(x, y) * I(x, y)|
$$

In the above formula:

* - The convolution operation;

$\nabla$ _ Two-dimensional differential operator of Laplace

$\nabla \equiv(\partial / \partial x, \partial / \partial y)$

$$
G(x, y)=\frac{1}{2 \pi \sigma^{2}} e^{\frac{\left(x-x_{0}\right)^{2}+\left(y-y_{0}\right) 2}{2 \sigma^{2}}}
$$

The two-dimensional Gaussian function are used for image convolution smoothing, and then differential is obtained along the horizontal direction and the vertical direction to get the grayscale gradient image. The gradient threshold is set to obtain the edge point set $\left(x_{j}, y_{j}\right), \quad j=1, \ldots, n$, as shown in Figure 2 (a).

Step two: voting is realized based on edge point set by the Hough transform. The basic strategy of the Hough transform is possible trajectories based on image space points in the parameter space to calculate the computed parameter point of duality (also known as the reference point), and cumulate a number of parameter points, which can be used to test various kinds of curves known, and then connect the curve point. Generally, the inner 
edge and the outer edge of the iris are round. In binaryzation edge image determines the inner and outer edge parameters of the iris by Hough transform, and Hough transform is defined as:

$$
H\left(x_{c}, y_{c}, r\right)=\sum_{j=1}^{n} h\left(x_{j}, y_{j}, x_{c}, y_{c}, r\right)
$$

In the above formula,

$$
\begin{aligned}
& h\left(x_{j}, y_{j}, x_{c}, y_{c}, r\right)=\left\{\begin{array}{cc}
1, & g\left(x_{j}, y_{j}, x_{c}, y_{c}, r\right)=0 \\
0, & \text { other }
\end{array}\right. \\
& g\left(x_{j}, y_{j}, x_{c}, y_{c}, r\right)=\left(x_{j}-x_{c}\right)^{2}+\left(y_{j}-y_{c}\right)^{2}-r^{2}
\end{aligned}
$$

Advantages of the Hough transform are suitable to detect a target of the known shape, which has high localization accuracy and less affected by noise and continuous curve and is not sensitive to the local light spot for the target with the clear edge obtained by the edge extraction and the binaryzation method. The shortage is that the Hough transform is based on the binaryzation boundary point method. When the image contrast is weak and the edge is blurry, it is necessary to choose the low threshold value [7]. Otherwise, it is not possible to extract the edge point accurately. In terms of the localization speed, Hough transform is performed in 3D space, and its computational complexity is very high, with the occupation of the large memory and the parameters extracted is limited by the quantization interval of the parameter space constraints. In addition, the effect is also affected by the performance of the edge detection operator.

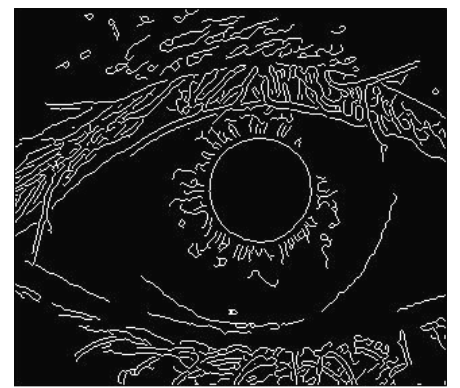

(a) The two-value edge image

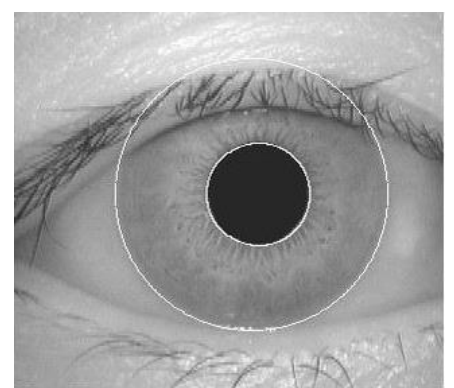

(b) The location results

Figure 2. Iris Localization based on Hough Transform

\subsection{The Method based on Geometric Features}

Localization method based on geometric features is employed to detect the iris by using the properties of the iris edge being similar to the circle, and circle geometrical features as well as properties of intersecting chord in the inner circle. The specific process is shown in the following. First, according to the features of the overall grayscale distribution of the iris image, the edge detection operator is used to extract the edge of the iris edge. Then, three edge points are found: $D_{1}\left(x_{1}, y_{1}\right), D_{2}\left(x_{2}, y_{2}\right), D_{3}\left(x_{3}, y_{3}\right)$. In Figure 3 , any two points among three points are combined as a string within the circle, which intersects $O_{1}$ with the perpendicular bisector of the string. Next, it is necessary to find out a few different points obtained by this method to search for a series of intersection $\mathrm{O}_{2}$. $\mathrm{O}_{3} \ldots$ The average value is calculated to coordinate of a series of intersection points, which means the average value determines $O$ the center of the iris edge[8], as shown in Figure 3. The key step of the algorithm is to find accurately the boundary points. The localization speed of the method is greatly influenced by the quality of the image. When the image quality is not high, the performance of the algorithm will drop sharply. 


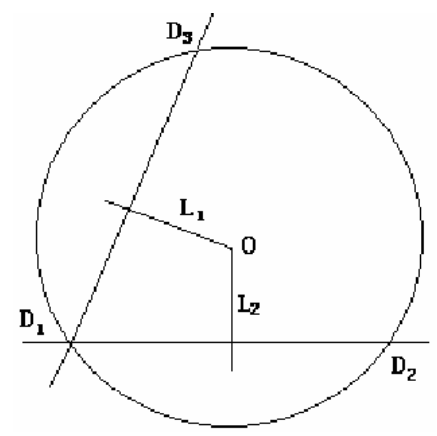

Figure 3. Iris Location Method based on Geometric Features

\section{The Rough Localization of the Iris Inner Edge}

The pupil shape is approximated as a circle, as shown in Figure 4. Four tangent lines of the pupil is constructed based on the image processing common coordinate, and four tangential points of the pupil are: $M\left(x_{1}, y_{1}\right), N\left(x_{2}, y_{2}\right), P\left(x_{3}, y_{3}\right), Q\left(x_{4}, y_{4}\right)$. The rough localization center of the pupil edge $\left(x_{\mathrm{p}}, y_{\mathrm{p}}\right)$ is the square center of the pupil external tangent determined by formula (13). The radius $r_{\mathrm{p}}$, regards as the mean of tangent distance determined by formula (14).

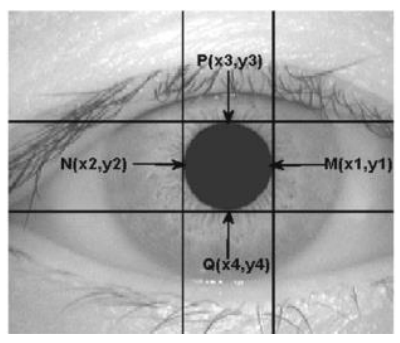

Figure 4. Localization Principle for Pupil Edge

$$
\begin{array}{r}
\left\{\begin{array}{c}
x_{p}=\frac{x_{3}+x_{4}}{2} \\
y_{p}=\frac{y_{1}+y_{2}}{2}
\end{array}\right. \\
\left\{\begin{array}{c}
r_{p}=\frac{r_{1}+r_{2}}{2} \\
r_{1}=\frac{x_{4}-x_{3}}{2} \\
r_{2}=\frac{y_{1}-y_{2}}{2}
\end{array}\right.
\end{array}
$$

The principle of tangency points localization of the pupil is shown in Figure 5. Circle $O$ represents the pupil area. the iris image is scaned by interval $\Delta n$. When the number of pixels in a certain row whose grayscale value equal grayscale peak $g_{\text {pupil }}$ reaches $N$, the endpoints of a string $L_{1}^{\prime}$ and $L_{2}^{\prime}$ are roughly localized in the circular $O$. Because of the existence of grayscale transition with the inner edge of the iris, $L_{1}^{\prime}$ and $L_{2}^{\prime}$ are often not the true boundary points, and it is possible for the strings $L_{1}^{\prime} L_{2}^{\prime}$ to have gray mutation points. For precise localization of the boundary point $L_{1}^{\prime}$ and $L_{2}^{\prime}$, the pixel point search is performed from the center points of the strings $L_{1}^{\prime} L_{2}^{\prime}$ along the line to the two end point of the current pixel. When a pixel gray value and the average value of the outer end of adjacent four pixel points grayscale are all larger than the threshold value $g_{\text {thresh }}$, it is shown that the true boundary points $L_{1}$ and $L_{2}$ are found. Perpendicular bisectors of $L_{1}$ and $L_{2}$ combine the pupil edge at the points $P, Q$, to find a couple of the tangency points of the 
pupil. According to the above principle, the iris image from different directions is respectively to implement the row-column scanning, and finally get four couples of the tangent points. Half of the mean of the tangent distance is used as a rough localization radius of the pupil, and the mean of $P, Q$ coordinates is regarded as the rough localization coordinates of the pupil.

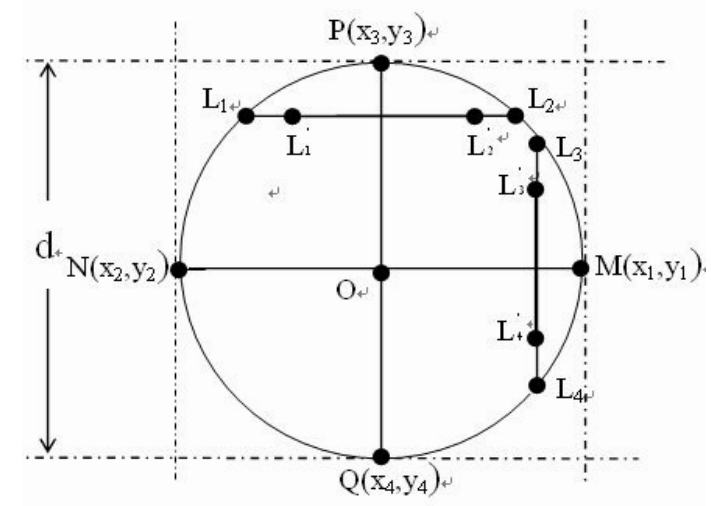

Figure 5. Localizing Point of Tangency in Pupil

Mathematical description of the above process is shown as follows:

(1)To find the initial boundary point.

The iris image is implement row scan by interval $\Delta n$, finding value $m$ to satisfy the first row in formula 15. According to formula 16, the center points of the strings $L_{1}^{\prime} L_{2}^{\prime}$ are localized as $L_{m}^{\prime}\left(m, y_{m}^{\prime}\right)$.

$$
\begin{gathered}
\operatorname{numel}\left(I(m, y)=g_{\text {pupil }}\right) \geq N \\
y_{m}^{\prime}=\frac{\sum_{i=1}^{n_{1}} y_{i}^{\prime}}{n_{1}}
\end{gathered}
$$

$I(m, y)$ represents the grayscale function of the image, while numel is to find quantity function. $y_{I}$ in the m line is all $y$ to meet formula (14).

(2) To determine the true boundary point.

For precise positioning of the boundary points $L_{1}$ and $L_{2}$, it is necessary to search from the centre point $L_{m}^{\prime}$ along line $m$ respectively to the two ends of the points. When formulas (15) and (16) are simultaneously satisfied, the boundary points of $L_{l}\left(m, y_{5}\right), L_{2}\left(m, y_{6}\right)$ are determined.

$$
\begin{aligned}
& \left\{\begin{array}{c}
I\left(m, y_{5}\right) \geq g_{\text {thresh }} \\
I\left(m, y_{6}\right) \geq g_{\text {thresh }}
\end{array}\right. \\
& \left\{\begin{array}{c}
\frac{\sum_{i=0}^{4} I\left(m, y_{5}-i\right)}{5} \geq g_{\text {thresh }} \\
\end{array}\right.
\end{aligned}
$$




$$
\frac{\sum_{i=0}^{4} I\left(m, y_{6}+i\right)}{5} \geq g_{\text {thresh }}
$$

The parameter $y_{m}$ of the center point $L_{m}\left(m, \mathrm{y}_{m}\right)$ of the strings $L_{1} L_{2}$ is determined by:

$$
y_{m}=\frac{\sum_{i=1}^{n_{2}} y_{i}}{n_{2}}
$$

$y_{i}$ is $y$ of $y_{I}^{\prime}$ and meeting $I(m, y) \leq g_{\text {pupil }}$.

(3) To localization points of tangency.

The search is done from the center point $L_{m}$ along the $y_{m}$ column respectively to the upper and lower end points to set in $P\left(x_{3}, y_{3}\right)$ and $Q\left(x_{4}, y_{4}\right)$ with the pupil edge. When the points of tangency meet first formulas (18), (19), $P$ and $Q$ points are determined, while $y_{3}$ and $y_{4}$ is determined by formula (20).

$$
\begin{aligned}
& \left\{\begin{array}{c}
I\left(x_{3}, y_{m}\right) \geq g_{\text {thresh }} \\
I\left(x_{4}, y_{m}\right) \geq g_{\text {thresh }}
\end{array}\right. \\
& \left\{\begin{array}{c}
\frac{\sum_{i=0}^{4} I\left(x_{3}-i, y_{m}\right)}{5} \geq g_{\text {thresh }} \\
\sum_{i=0}^{4} I\left(x_{4}+i, y_{m}\right) \\
5 \\
y_{3}=y_{4}=y_{m}
\end{array}\right.
\end{aligned}
$$

For the rough localization to the pupils, value of $N$ cannot be too small to prevent falling into the inner edge transition zone and influencing the localization accuracy by the noise outside the pupil, where $N$ is set as 30 . General pupil grayscale value is less than 80[9], and therefore the $g_{\text {thresh }}$ is set as 80 .

\section{Accurate Localization of the Iris Edge}

\subsection{Accurate Localization of the Inner Edge}

As the pupil edge is obviously darker than surrounding area, and therefore the calculus method of Daugman is suitable for localization. $\left(x_{p}, y_{p}, r_{p}\right)$ is regarded as the rough localization results of pupil edge parameters. In order to improve the speed of iris inner edge localization, the search range of the pupil edge parameters is limited, and the search range of the pupil edge center is limited in $\left(x_{p}, y_{p}\right)$ as the center, the size of $25 * 25$ neighboring area. The variation range of radius is limited in the interval $\left[r_{p}-25, r_{p}+25\right]$, and finally the accurate parameters of the inner edge is obtained as $\left(X_{P}, Y_{P}, R_{P}\right)$.

$$
\max _{\left(r, x_{0}, y_{0}\right)}\left|G_{\sigma}(R) * \frac{\partial}{\partial R} \iint_{D} \frac{I(x, y)}{\pi R^{2}} d \delta\right|
$$

\subsection{Accurate Localization of the Outer Edge}

The transition zone outer edge of the iris is usually wide and grayscale change in the outer edge is not stronger than the inner edge. In addition, the rich texture information of 
iris (such as nerve ring) will also affect the iris outer edge localization. The direct application of Daugman line integral method sometimes cannot accurately localize the iris outer edge. The surface integral is used in the proposed algorithm instead of the above line integral for the outer edge localization. Finally, the outer edge parameters $\left(X_{\mathrm{I}}, Y_{\mathrm{J}}, R_{\mathrm{I}}\right)$ are obtained.

\section{The Results and Analysis of the Experiments}

The experiment selects CASIA V2.0 iris database [10], and each eye randomly selects an image, and there are a total of 108 iris images. The proposed algorithm, the calculus method and Hough transform method are respectively used for localization experiment. An iris image disturbed by the eyelid and eyelash is chosen. The localization results are shown in Figure 6. In Table 1, the localization parameters of the above three kinds of algorithm are listed.

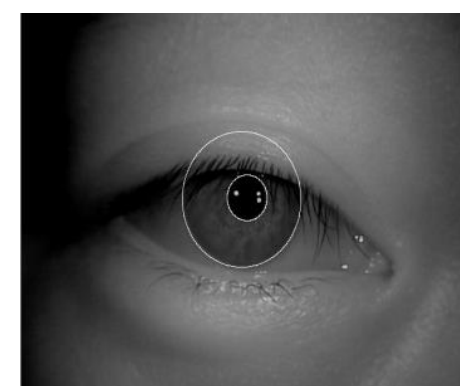

(a) The Proposed Algorithm

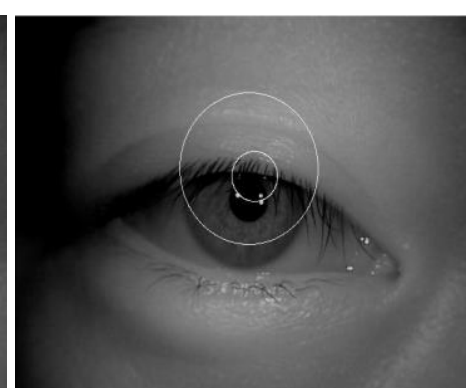

(b) Daugman's Algorithm

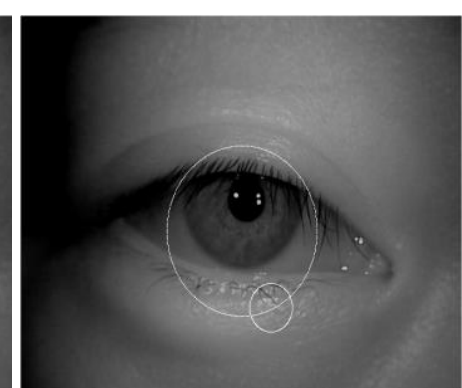

(c) Hough Transform

Figure 6. Iris Localization under Asymmetrical Quality of Illumination

Table 1. Localization Parameters with the Three Kinds of Algorithms

\begin{tabular}{cccccccc}
\hline \multirow{2}{*}{ Algorithm } & \multicolumn{3}{c}{ Parameters of inner edge } & \multicolumn{3}{c}{ Parameters of inner edge } \\
\cline { 2 - 8 } & $X_{P}$ & $Y_{P}$ & $R_{P}$ & $X_{I}$ & $Y_{I}$ & $R_{I}$ \\
\hline Wildes & 148 & 176 & 50 & 154 & 198 & 84 \\
Daugman & 148 & 176 & 50 & 141 & 177 & 102 \\
The proposed algorithm & 148 & 176 & 49 & 141 & 177 & 102 \\
\hline
\end{tabular}

It can be seen from the experimental results that the accuracy rate of localization by this algorithm is slightly higher than that of the classical algorithm of iris localization, and the localization performance is superior to the classical algorithm for the following reasons:

(1) The geometric features of the circle are used to localize the pupil. The localization process is linear, and the time complexity is $O(n)$, to avoid the floating-point arithmetic of the classic localization algorithms.

(2) The prior knowledge is made full use of to reduce the search range of the outer edge of the center of the calculus method. Furthermore, the proposed algorithm combines the calculus method for precise localization, to ensure the accuracy and improve the localization speed.

From the concrete performance of the localization, the boundary of the inner edge is obvious, and therefore the three algorithms can localize accurately; the boundary of the 
outer edge is not obvious, and therefore the localization of Hough transform is in failure which is interfered by eyelash factors.

\section{Conclusion}

The results of the experiment have shown that the proposed algorithm first uses the circular geometry feature to get a fast rough localization for inner iris edges, and then use the calculus method to precisely localize the inner iris edges. The coupling of the internal and external edges of iris is used to narrow searching range edge of the iris center of the calculus method. Based on the above algorithm, the search range of differential operator is greatly reduced. The test results have shown that localization results to over 100 iris images of CASIA (version 2.0) show that the proposed algorithm is accurate and robust.

\section{Acknowledgements}

The study was supported by following Funds.

(1) scientific research fund of henan provincial education department[14A520085].

(2)Teacher education curriculum reform of Henan Province [2014-JSJYYB-026].

(3) Youth Science Fund of Henan Normal University.

\section{References}

[1] J. Daugman, "How iris recognition works", IEEE Transactions on Circuits and Systems for Video Technology, vol. 14, no. 21, (2004), pp. 1050-1052.

[2] R. Wildes, "Iris recognition: an emerging biometric technology", Proceeding of the IEEE, vol.85, (1997), pp. $1348-1363$.

[3] Q. C. Tian and R. S. Zhang, "Review of biological feature recognition", Application Research of computers, vol. 26, no. 12,(2009),p. 4404.

[4] C. Zhang, X. Y. Yuan and P. F. Shi, "Fast localization algorithm of iris inner and outer edge", computer engineering application, vol. 27, (2005), pp. 60-61.

[5] J. H. Wu, D. X. Zou and J. H. Li, "A fast and accurate algorithm for iris localization, Chinese Journal of scientific instrument, vol. 28,no. 8, (2007), p. 1470.

[6] M. Li, T. N. Tan and Y. H. Wang, "Efficient Iris Recognition by Characterizing Key Local Variations", IEEE Transactions on Image Processing, vol. 13, no. 6, (2004), pp. 738-749.

[7] Z. Ma and W. Huang, "Curve fitting algorithm for iris localization research", Journal of University of Electronic Science and technology, vol. 38, no. 3, (2009), pp. 428-430.

[8] Z. X. Wang and W. S. Li, "iris location method based on improved active contour line", Computer engineering and design, vol. 30, no. 10, (2009), pp. $53-258$.

[9] B. Xue, W. Y. Liu, J. T. Wang and K. L. Zuo, "Preprocessing algorithm for iris image", Journal of Optoelectronics laser, vol. 14, no. 7, (2003), pp. 742-745.

[10] Institute of Automation, Chinese Academy of Sciences, CASIA iris image database (ver 2.0)[DB].http://www.sinobiometrics.com.2004

\section{Authors}

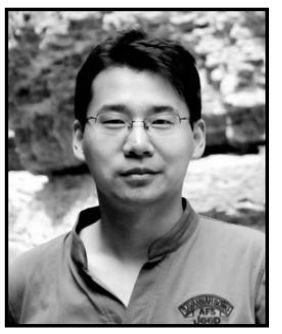

Ming Fei Wang, he received her Master Degree from Henan Normal University in 2007.He is now an Lecturer of Henan Mechanical and Electrical Engineering College in China. His research interests include computer application, Computer Network. He has published more than 6 papers in journals and conferences. 


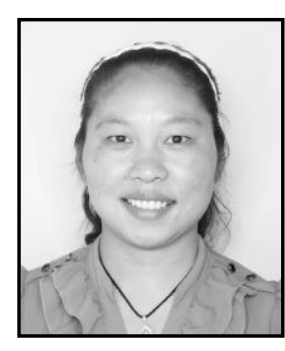

Rui Yun Xie, he received her Master Degree from Wuhan University of Technology in 2009. She is now a Lecturer of Henan Mechanical and Electrical Engineering College in China. Her research interests include computer application, Computer Network. She has published more than 5 papers in journals and conferences.

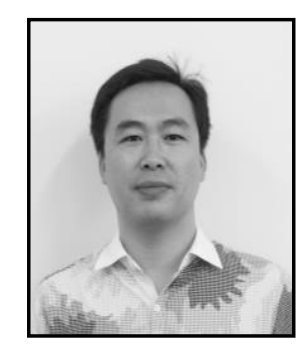

BenZhai Hai, he received his Master Degree from Wuhan University of Technology in 2009. He is now a Lecturer of Henan Normal University in China. His research interests include computer application; Computer Network. He has published more than 20 papers in journals and conferences. 